\title{
A Remarkably Efficient Coupling of Acid Chlorides with Alkynes in Water
}

\author{
Liang Chen ${ }^{a}$ and Chao-Jun $\mathrm{Li}^{\star a, b}$ \\ ${ }^{a}$ : Department of Chemistry, Tulane University, New Orleans, Louisiana 70118 USA; ${ }^{b}$ Department of \\ Chemistry, McGill University, 801 Sherbrooke St. West, Montreal, Quebec H3A 2K6, Canada. \\ Email:cj.li@mcgill.ca
}

\section{Supporting information}

\section{Experimental:}

${ }^{1} \mathrm{H}$ NMR, ${ }^{13} \mathrm{C}$ NMR, GC-MS and IR were performed at the Department of Chemistry of Tulane University.

General Procedure. All reactions were carried out by the following general procedure except the synthesis of compound (5), 1-(4-nitrophenyl)-3-phenylprop-2-yn-1-one. 1 mmol of terminal alkynes, $2 \mathrm{mmol}$ of acid chloride, $20 \mathrm{mg}$ of sodium lauryl sulfate (7 mol\%), $14 \mathrm{mg} \mathrm{PdCl}\left(\mathrm{PPh}_{3}\right)_{2}(2 \mathrm{~mol} \%)$ and $10 \mathrm{mg} \mathrm{CuI} \mathrm{(5} \mathrm{mol \% )} \mathrm{were} \mathrm{added} \mathrm{in} \mathrm{a} \mathrm{small}$ test tube $(10 \mathrm{~mL})$ that was cooled by ice-water bath then $1 \mathrm{~mL}$ ice-cold water that dissovled $414 \mathrm{mg} \mathrm{K}_{2} \mathrm{CO}_{3}(3 \mathrm{mmol})$ was added to the test tube in samll portions while stirring. After the addition was completed, the reaction mixture was capped, then heated and stirred at $65^{\circ} \mathrm{C}$ for 4 hours. Then the reaction mixture was cooled and extracted with ethyl ether $(3 \times 10 \mathrm{~mL})$ and concentrated in vacuo. The residue was purified by silica gel column chromatography (hexane : ethyl acetate $=10: 1$ or $20: 1$ ) to give the pure product. 


\section{Characteristic Data}

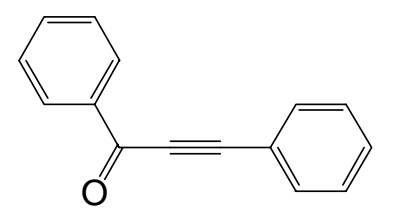

1,3-diphenylprop-2-yn-1-one

1,3-diphenylprop-2-yn-1-one (1) ${ }^{\mathbf{1}}(202 \mathrm{mg}, 98 \%$ yield $){ }^{1} \mathrm{H}$ NMR $\left(400 \mathrm{MHz}, \mathrm{CDCl}_{3}\right): \delta$ $8.19(\mathrm{dd}, J=8.0 \mathrm{~Hz}, 1.6 \mathrm{~Hz}, 2 \mathrm{H}), 7.63(\mathrm{~d}, J=8.0 \mathrm{~Hz}, 2 \mathrm{H}), 7.57(\mathrm{t}, J=8.0 \mathrm{~Hz}, 1 \mathrm{H}), 7.48-$ $7.34(\mathrm{~m}, 5 \mathrm{H}) ;{ }^{13} \mathrm{C}$ NMR (400 MHz, $\left.\mathrm{CDCl}_{3}\right): \delta 178.2,137.0,134.4,133.3,131.1,129.8$, 129.0, 128.9, 120.2, 93.4, 87.2; IR (neat) $\mathrm{cm}^{-1}$ 3057, 2199, 1640; GC-MS (Relative Intensity) $206\left(\mathrm{M}^{+}, 70\right), 178$ (100), 129 (78), 101 (12), 75 (22), 51 (19).

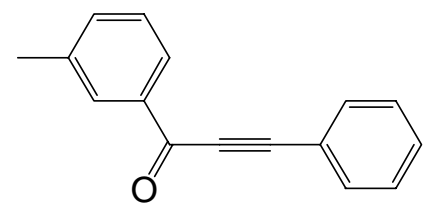

3-phenyl-1-m-tolylprop-2-yn-1-one

3-phenyl-1-m-tolyprop-2-yn-1-one (2) ${ }^{2}$ (218 mg, 99\% yield) ${ }^{1} \mathrm{H}$ NMR (400 MHz, $\left.\mathrm{CDCl}_{3}\right): \delta 8.04-7.99(\mathrm{~m}, 2 \mathrm{H}), 7.68-7.66(\mathrm{~m}, 2 \mathrm{H}), 7.47-7.39(\mathrm{~m}, 5 \mathrm{H}), 2.43(\mathrm{~s}, 3 \mathrm{H}) ;{ }^{13} \mathrm{C}$ NMR (400 MHz, $\left.\mathrm{CDCl}_{3}\right): \delta 178.5,138.7,137.1,135.2,133.3,131.0,130.0,128.9,128.7$, 127.4, 120.4, 93.1, 87.2, 21.6; IR (neat) $\mathrm{cm}^{-1} 3057,2914,2205$, 1633; GC-MS (Relative Intensity) 220 (M+ , 69), 192 (100), 129 (73), 101 (17), 91 (12), 75 (28), 65 (19), 51 (15).

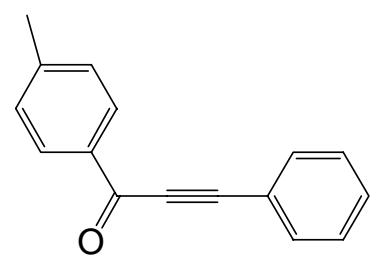

3-phenyl-1-p-tolylprop-2-yn-1-one 
3-phenyl-1-p-tolyprop-2-yn-1-one (3) ${ }^{\mathbf{1}}$ (215 mg, 98\% yield) ${ }^{1} \mathrm{H}$ NMR (400 MHz, $\left.\mathrm{CDCl}_{3}\right): \delta 8.07(\mathrm{~d}, J=8.4 \mathrm{~Hz}, 2 \mathrm{H}), 7.62(\mathrm{~d}, J=7.6 \mathrm{~Hz}, 2 \mathrm{H}), 7.42-7.35(\mathrm{~m}, 3 \mathrm{H}), 7.25(\mathrm{~d}, J$ $=8.4 \mathrm{~Hz}, 2 \mathrm{H}), 2.37(\mathrm{~s}, 3 \mathrm{H}) ;{ }^{13} \mathrm{C} \mathrm{NMR}\left(400 \mathrm{MHz}, \mathrm{CDCl}_{3}\right): \delta 177.9,145.5,134.8,133.3$, 131.0, 129.9, 129.6, 128.9, 120.4, 92.9, 87.2, 22.1; IR (neat) $\mathrm{cm}^{-1} 3057,2914,2192,1640$; GC-MS (Relative Intensity) 220 (M+ , 77), 192 (100), 191 (54), 129 (50), 101 (8), 75 (12), $51(5)$.

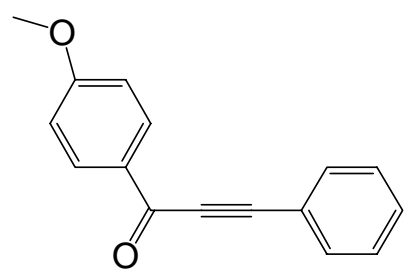

1-(4-methoxyphenyl)-3-phenylprop-2-yn-1-one

1-(4-methoxyphenyl)-3-phenylprop-2-yn-1-one (4) ${ }^{\mathbf{3}}$ (193 mg, 82\% yield) ${ }^{1} \mathrm{H}$ NMR $\left(400 \mathrm{MHz}, \mathrm{CDCl}_{3}\right): \delta 8.09(\mathrm{~d}, J=8.8 \mathrm{~Hz}, 2 \mathrm{H}), 7.56(\mathrm{~d}, J=7.6 \mathrm{~Hz}, 2 \mathrm{H}), 7.32-7.28(\mathrm{~m}$, $3 \mathrm{H}), 6.87(\mathrm{~d}, J=8.8 \mathrm{~Hz}, 2 \mathrm{H}), 3.76(\mathrm{~s}, 3 \mathrm{H}) ;{ }^{13} \mathrm{C} \mathrm{NMR}\left(400 \mathrm{MHz}, \mathrm{CDCl}_{3}\right): \delta 176.6,164.5$, 132.9, 131.9, 130.6, 130.2, 128.6, 120.2, 113.8, 92.3, 86.9, 55.5; IR (neat) $\mathrm{cm}^{-1} 3057$, 2199, 1627; GC-MS (Relative Intensity) 236 (M+ , 79), 208 (100), 193 (92), 165 (65), 129 (53), 101 (17), 75 (28), 63 (24), 51 (13).

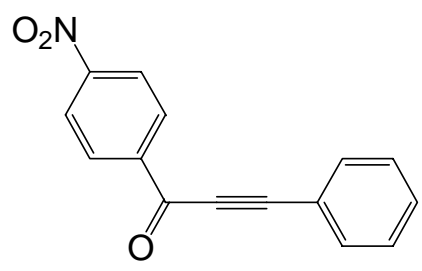

1-(4-nitrophenyl)-3-phenylprop-2-yn-1-one

1-(4-nitrophenyl)-3-phenylprop-2-yn-1-one (5) ${ }^{3}$ (128 mg, 51\% yield) 4-nitrobenzoyl chloride (370 mg, $2 \mathrm{mmol}, 2$ equiv) was dissolved in $200 \mathrm{mg}$ toluene in a small test tube $(10 \mathrm{~mL})$ that was cooled by ice-water bath, then phenylacetylene $(102 \mathrm{mg} .1 \mathrm{mmol}, 1$ 
equiv), sodium lauryl sulfate (20 mg, $7 \mathrm{~mol} \%), \mathrm{PdCl}_{2}\left(\mathrm{PPh}_{3}\right)_{2}(14 \mathrm{mg}, 2 \mathrm{~mol} \%)$ and $\mathrm{CuI}$ (10 mg, $5 \mathrm{~mol} \%$ ) were added into the test tube. After that, $1 \mathrm{~mL}$ ice-cold water that dissovled $\mathrm{K}_{2} \mathrm{CO}_{3}$ (414 $\mathrm{mg}, 3 \mathrm{mmol}$ ) was added to the test tube in samll portions while stirring. After completing the addition, the reaction mixture was capped, then heated and stirred at $65^{\circ} \mathrm{C}$ for 4 hours. Then the reaction mixture was cooled and extracted with ethyl ether $(3 \times 10 \mathrm{~mL})$ and concentrated in vacuo. The residue was purified by silica gel column chromatography (hexane : ethyl acetate $=10: 1$ ) to give $128 \mathrm{mg}$ of pure product 1 (4-nitrophenyl)-3-phenylprop-2-yn-1-one (51\% yield). ${ }^{1} \mathrm{H} \mathrm{NMR}\left(400 \mathrm{MHz}, \mathrm{CDCl}_{3}\right): \delta$ $8.36(\mathrm{~s}, 4 \mathrm{H}), 7.70(\mathrm{~d}, J=8.4 \mathrm{~Hz}, 2 \mathrm{H}), 7.52-7.42(\mathrm{~m}, 3 \mathrm{H}) ;{ }^{13} \mathrm{C}$ NMR $\left(400 \mathrm{MHz}, \mathrm{CDCl}_{3}\right)$ : $\delta 176.1,151.1,141.2,133.5,131.7,130.7,129.1,124.1,119.6,95.7,86.7$; IR (neat) $\mathrm{cm}^{-1}$ 3057, 2186, 1646; GC-MS (Relative Intensity) $252\left(\mathrm{M}^{+}, 25\right), 223$ (27), 129 (100).

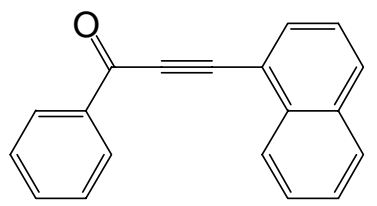

3-(naphthalen-1-yl)-1-phenylprop-2-yn-1-one

3-(naphthalen-1-yl)-1-phenylprop-2-yn-1-one (6) ${ }^{\mathbf{4}}$ (205 mg, 80\% yield) ${ }^{1} \mathrm{H}$ NMR (400 $\left.\mathrm{MHz}_{\mathrm{CDCl}}\right): \delta 8.40(\mathrm{~d}, J=8.0 \mathrm{~Hz}, 1 \mathrm{H}), 8.32(\mathrm{~d}, J=8.0 \mathrm{~Hz}, 2 \mathrm{H}), 7.88(\mathrm{~d}, J=8.0 \mathrm{~Hz}$, 2H), $7.81(\mathrm{~d}, J=8.0 \mathrm{~Hz}, 1 \mathrm{H}), 7.60(\mathrm{t}, J=8.0 \mathrm{~Hz}, 2 \mathrm{H}), 7.51(\mathrm{t}, J=8.0 \mathrm{~Hz}, 3 \mathrm{H}), 7.41(\mathrm{t}, J=$ $8.0 \mathrm{~Hz}, 1 \mathrm{H}) ;{ }^{13} \mathrm{C} \mathrm{NMR}\left(400 \mathrm{MHz}, \mathrm{CDCl}_{3}\right): \delta 178.2,137.3,134.5,133.9,133.6,133.3$, $131.9,129.9,129.0,128.9,128.1,127.3,126.0,125.5,117.8,92.0,91.8 ;$ IR (neat) $\mathrm{cm}^{-1}$ 3057, 2186, 1640; GC-MS (Relative Intensity) 256 (M+, 96), 227 (100), 179 (63), 150 (46), 77 (28), 51 (27). 


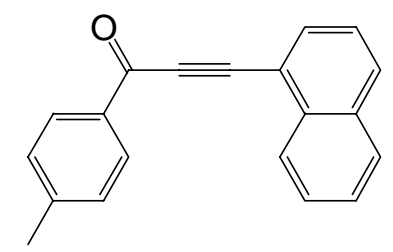

3-(naphthalen-1-yl)-1-p-tolylprop-2-yn-1-one

3-(naphthalen-1-yl)-1-p-tolylprop-2-yn-1-one (7) $)^{\mathbf{5}}$ (262 mg, 97\% yield) ${ }^{1} \mathrm{H}$ NMR (400 $\left.\mathrm{MHz}, \mathrm{CDCl}_{3}\right): \delta 8.40(\mathrm{~d}, J=8.4 \mathrm{~Hz}, 1 \mathrm{H}), 8.19(\mathrm{~d}, J=8.4 \mathrm{~Hz}, 2 \mathrm{H}), 7.93-7.90(\mathrm{~m}, 2 \mathrm{H})$, $7.85(\mathrm{~d}, J=8.4 \mathrm{~Hz}, 1 \mathrm{H}), 7.62(\mathrm{t}, J=7.2 \mathrm{~Hz}, 1 \mathrm{H}), 7.54(\mathrm{t}, J=7.2 \mathrm{~Hz}, 1 \mathrm{H}), 7.46(\mathrm{t}, J=7.2$ $\mathrm{Hz}, 1 \mathrm{H}), 7.31(\mathrm{~d}, J=8.0 \mathrm{~Hz}, 2 \mathrm{H}), 2.42(\mathrm{~s}, 3 \mathrm{H}) ;{ }^{13} \mathrm{C} \mathrm{NMR}\left(400 \mathrm{MHz}, \mathrm{CDCl}_{3}\right): \delta 177.9$, $145.5,135.0,133.9,133.4,133.3,131.7,130.0,129.7,128.8,128.0,127.2,126.0,125.5$, 118.0, 92.0, 91.3, 22.1; IR (neat) $\mathrm{cm}^{-1} 3058,2917,2186,1635$; GC-MS (Relative Intensity) $270\left(\mathrm{M}^{+}, 100\right), 242$ (88), 179 (43), 150 (28), 121 (15), 91 (9).

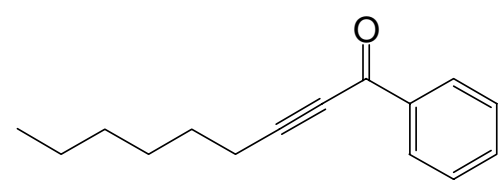

1-phenylnon-2-yn-1-one

1-phenylnon-2-yn-1-one (8) ${ }^{6}\left(141 \mathrm{mg}, 66 \%\right.$ yield) ${ }^{1} \mathrm{H}$ NMR (400 MHz, $\left.\mathrm{CDCl}_{3}\right): \delta 8.09$ $(\mathrm{d}, J=8.0 \mathrm{~Hz}, 2 \mathrm{H}), 7.54(\mathrm{t}, J=7.6 \mathrm{~Hz}, 1 \mathrm{H}), 7.42(\mathrm{t}, J=8.0 \mathrm{~Hz}, 2 \mathrm{H}), 2.44(\mathrm{t}, J=6.8 \mathrm{~Hz}$, $2 \mathrm{H}), 1.64-1.58(\mathrm{~m}, 2 \mathrm{H}), 1.46-1.40(\mathrm{~m}, 2 \mathrm{H}), 1.29-1.25(\mathrm{~m}, 4 \mathrm{H}), 0.85(\mathrm{t}, J=6.8 \mathrm{~Hz}, 3 \mathrm{H})$; ${ }^{13} \mathrm{C}$ NMR (400 MHz, $\left.\mathrm{CDCl}_{3}\right): \delta 178.4,137.1,134.1,129.7,128.7,97.1,79.9,31.4,28.8$, 28.0, 22.7, 19.4, 14.2; IR (neat) $\mathrm{cm}^{-1} 3057,2927,2856,2192$, 1640; GC-MS (Relative Intensity) $214\left(\mathrm{M}^{+}, 5\right), 199$ (10), 186 (26), 171 (23), 157 (48), 145 (19), 129 (19), 115 (52), 105 (100), 77 (73), 51 (30). 


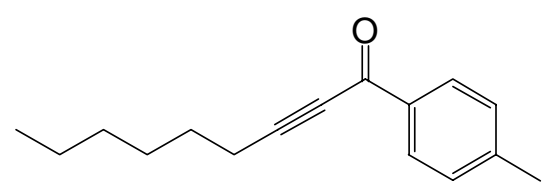

1-p-tolylnon-2-yn-1-one

1-p-tolylnon-2-yn-1-one (9) ${ }^{\mathbf{1}}\left(176 \mathrm{mg}, 77 \%\right.$ yield) ${ }^{1} \mathrm{H} \mathrm{NMR}\left(400 \mathrm{MHz}, \mathrm{CDCl}_{3}\right): \delta 7.98$ $(\mathrm{d}, J=8.0 \mathrm{~Hz}, 2 \mathrm{H}), 7.21(\mathrm{~d}, J=8.0 \mathrm{~Hz}, 2 \mathrm{H}), 2.43(\mathrm{t}, J=7.2 \mathrm{~Hz}, 2 \mathrm{H}), 2.36(\mathrm{~s}, 3 \mathrm{H}), 1.65-$ $1.57(\mathrm{~m}, 2 \mathrm{H}), 1.46-1.38(\mathrm{~m}, 2 \mathrm{H}), 1.29-1.25(\mathrm{~m}, 4 \mathrm{H}), 0.86(\mathrm{t}, J=7.2 \mathrm{~Hz}, 3 \mathrm{H}) ;{ }^{13} \mathrm{C} \mathrm{NMR}$ (400 MHz, $\left.\mathrm{CDCl}_{3}\right): \delta 178.1,145.1,134.8,129.9,129.4,96.5,79.9,31.4,28.8,28.0,22.7$, 22.0, 19.4, 14.2; IR (neat) $\mathrm{cm}^{-1} 3025,2934,2856,2186,1640$; GC-MS (Relative Intensity) $228\left(\mathrm{M}^{+}, 6\right), 213$ (25), 185 (63), 171 (42), 158 (20), 145 (19), 129 (26), 119 (100), 91 (39), 79 (11), 65 (10).

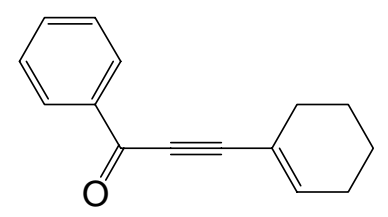

3-cyclohexenyl-1-phenylprop-2-yn-1-one

3-cyclohexenyl-1-phenylprop-2-yn-1-one (10) ${ }^{7}$ (174 mg, 83\% yield) ${ }^{1} \mathrm{H}$ NMR (400 $\left.\left.\mathrm{MHz}_{\mathrm{CDCl}}\right)\right): \delta 8.04(\mathrm{~d}, J=8.0 \mathrm{~Hz}, 2 \mathrm{H}), 7.48(\mathrm{~d}, J=7.2 \mathrm{~Hz}, 1 \mathrm{H}), 7.36(\mathrm{t}, J=8.0 \mathrm{~Hz}$, 2H), $6.46(\mathrm{~m}, 1 \mathrm{H}), 2.18-2.06(\mathrm{~m}, 4 \mathrm{H}), 1.59-1.50(\mathrm{~m}, 4 \mathrm{H}) ;{ }^{13} \mathrm{C} \mathrm{NMR}\left(400 \mathrm{MHz}, \mathrm{CDCl}_{3}\right)$ : $\delta 178.4,143.0,137.2,134.1,129.6,128.7,119.3,96.0,85.4,28.6,26.4,22.1,21.3$; IR (neat) $\mathrm{cm}^{-1} 3063,2927,2186,1633$; GC-MS (Relative Intensity) $210\left(\mathrm{M}^{+}, 82\right), 195$ (5), 181 (19), 167 (34), 153 (29), 133 (19), 115 (19), 105 (99), 77 (100), 51 (39).

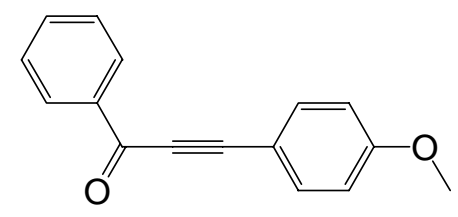

3-(4-methoxyphenyl)-1-phenylprop-2-yn-1-one 
3-(4-methoxyphenyl)-1-phenylprop-2-yn-1-one (11) ${ }^{\mathbf{8}}$ (234 mg, 99\% yield) ${ }^{1} \mathrm{H}$ NMR $\left(400 \mathrm{MHz}, \mathrm{CDCl}_{3}\right): \delta 8.08(\mathrm{~d}, J=8.0 \mathrm{~Hz}, 2 \mathrm{H}), 7.50-7.47(\mathrm{~m}, 3 \mathrm{H}), 7.37(\mathrm{t}, J=7.2 \mathrm{~Hz}$, $2 \mathrm{H}), 6.78(\mathrm{~d}, J=8.8 \mathrm{~Hz}, 2 \mathrm{H}), 3.68(\mathrm{~s}, 3 \mathrm{H}) ;{ }^{13} \mathrm{C} \mathrm{NMR}\left(400 \mathrm{MHz}, \mathrm{CDCl}_{3}\right): \delta 178.2,162.0$, 137.2, 135.4, 134.2, 129.7, 128.8, 114.7, 112.0, 94.7, 87.1, 55.6; IR (neat) $\mathrm{cm}^{-1} 3057$, 2956, 2192, 1635; GC-MS (Relative Intensity) 236 ( $\left.\mathrm{M}^{+}, 100\right), 208$ (52), 193 (64), 165 (55), 159 (91), 144 (20), 116 (25), 77 (50), 51 (41).

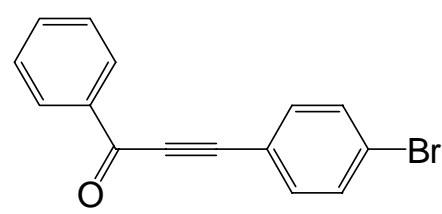

3-(4-bromophenyl)-1-phenylprop-2-yn-1-one

3-(4-bromophenyl)-1-phenylprop-2-yn-1-one (12) ${ }^{\mathbf{9}}$ (251 mg, 88\% yield) ${ }^{1} \mathrm{H}$ NMR (400 $\left.\mathrm{MHz}, \mathrm{CDCl}_{3}\right): \delta 8.06-8.03(\mathrm{~m}, 2 \mathrm{H}), 7.47-7.37(\mathrm{~m}, 7 \mathrm{H}) ;{ }^{13} \mathrm{C} \mathrm{NMR}\left(400 \mathrm{MHz}, \mathrm{CDCl}_{3}\right): \delta$ $177.9,136.8,134.6,134.5,132.3,129.8,128.9,125.8,119.2,91.9,87.9 ;$ IR (neat) $\mathrm{cm}^{-1}$ 3051, 2192, 1628; GC-MS (Relative Intensity) $286\left(\mathrm{M}^{+}+2,45\right), 284\left(\mathrm{M}^{+}, 46\right), 258$ (100), 256 (83), 206 (54), 176 (76), 151 (21), 128 (48), 101 (18), 77 (63), 51 (62).

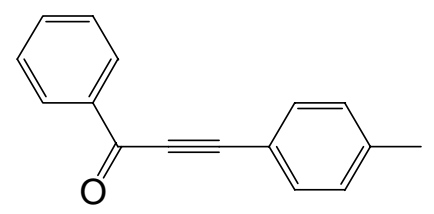

1-phenyl-3-p-tolylprop-2-yn-1-one

1-phenyl-3-p-tolylprop-2-yn-1-one (13) ${ }^{\mathbf{1 0}}\left(202 \mathrm{mg}, 92 \%\right.$ yield) ${ }^{1} \mathrm{H}$ NMR (400 MHz, $\left.\mathrm{CDCl}_{3}\right): \delta 8.18(\mathrm{dd}, J=8.4 \mathrm{~Hz}, 1.2 \mathrm{~Hz}, 2 \mathrm{H}), 7.57-7.44(\mathrm{~m}, 5 \mathrm{H}), 7.16(\mathrm{~d}, J=8.0 \mathrm{~Hz}, 2 \mathrm{H})$, $2.33(\mathrm{~s}, 3 \mathrm{H}) ;{ }^{13} \mathrm{C} \mathrm{NMR}\left(400 \mathrm{MHz}, \mathrm{CDCl}_{3}\right): \delta 178.2,141.8,137.1,134.3,133.4,129.7$, 129.7, 128.8, 117.1, 94.1, 87.0, 22.0; IR (neat) $\mathrm{cm}^{-1} 3063,2921,2199,1633$; GC-MS 
(Relative Intensity) 220 (M+ , 79), 192 (93), 191 (53), 143 (100), 115 (24), 89 (21), 77 (24), 63 (14), 51 (21).

1. Ahmed, M. S. M.; Mori, A. Org. Lett. 2003, 5, 3057.

2. Zanina, A. S.; Shergina, S. I.; Sokolov, I. E.; Kotlyarevskii, I. L. Seriya Khimicheskaya 1981, 5, 1158.

3. Alonso, D. A.; Najera, C.; Pacheco, M. C. J. Org. Chem. 2003, 69, 1615.

4. Delaude, L.; Masdeu, A. M.; Alper, H. Synthesis 1994, 11, 1149.

5. Kundu, N. G.; Pal, M.; Chowdhury, C. J. Chem. Res., Synop. 1995, 1, 4.

6. Parker, K. A.; Ledeboer, M. W. J. Org. Chem 1996, 61, 3214.

7. Marvell, E. N.; Gosink, T.; Churchley, P.; Li, T. J. Org. Chem. 1972, 37, 2989.

8. Fetter, J.; Nagy, I.; Giang, L. T.; Kajtar-Peredy, M.; Rockenbauer, A.; Korecz, L.; Czira, G. J. Chem. Soc., Perkin Trans. 1 2001, 9, 1131.

9. Qian, H.; Shao, L.-X.; Huang, X. Synlett 2001, 10, 1571.

10. Kakusawa, N.; Tobiyasu, Y.; Yasuike, S.; Yamaguchi, K.; Seki, H.; Kurita, J. Tetrahedron Lett. 2003, 44, 8589. 


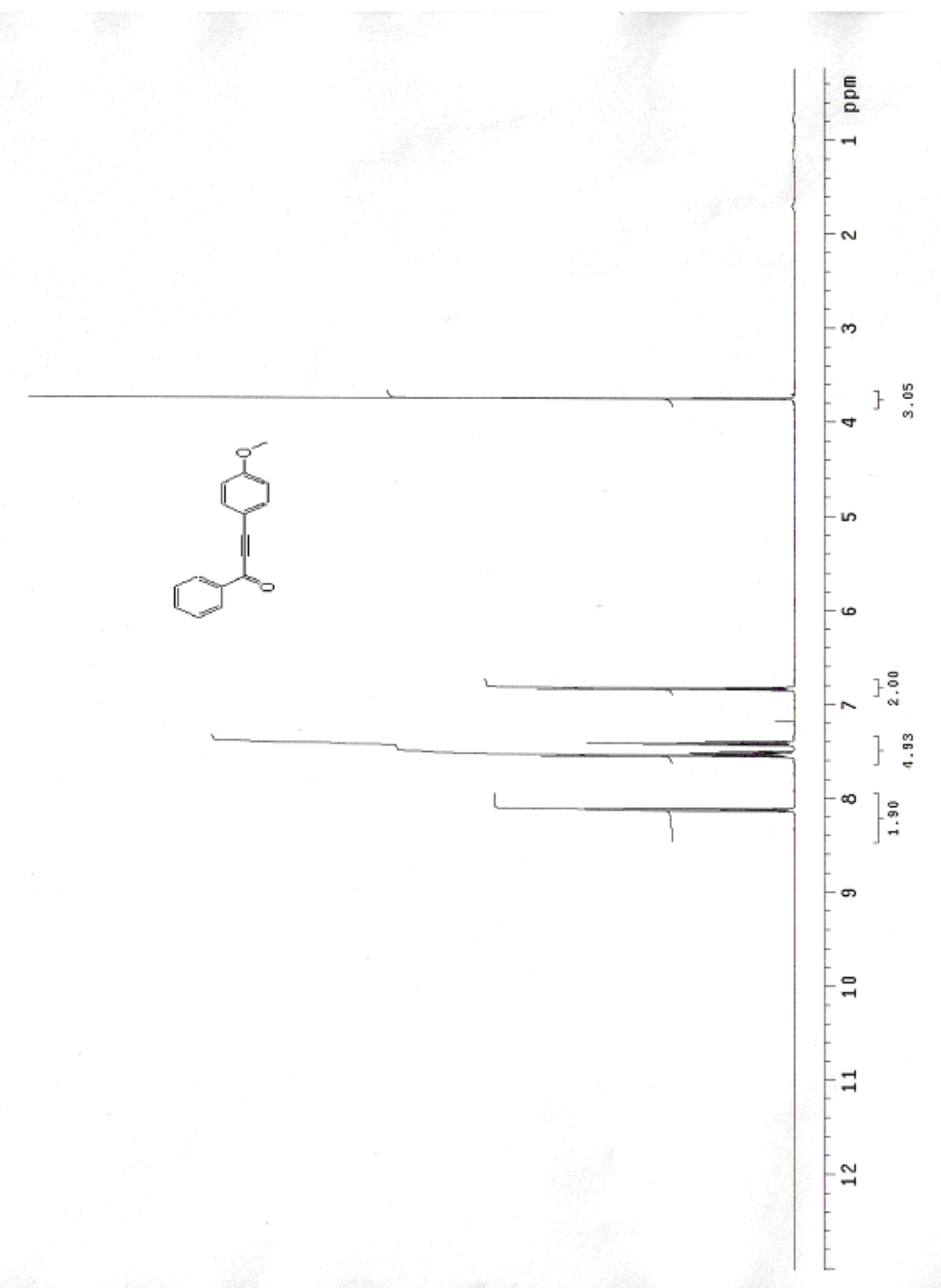

S-9 


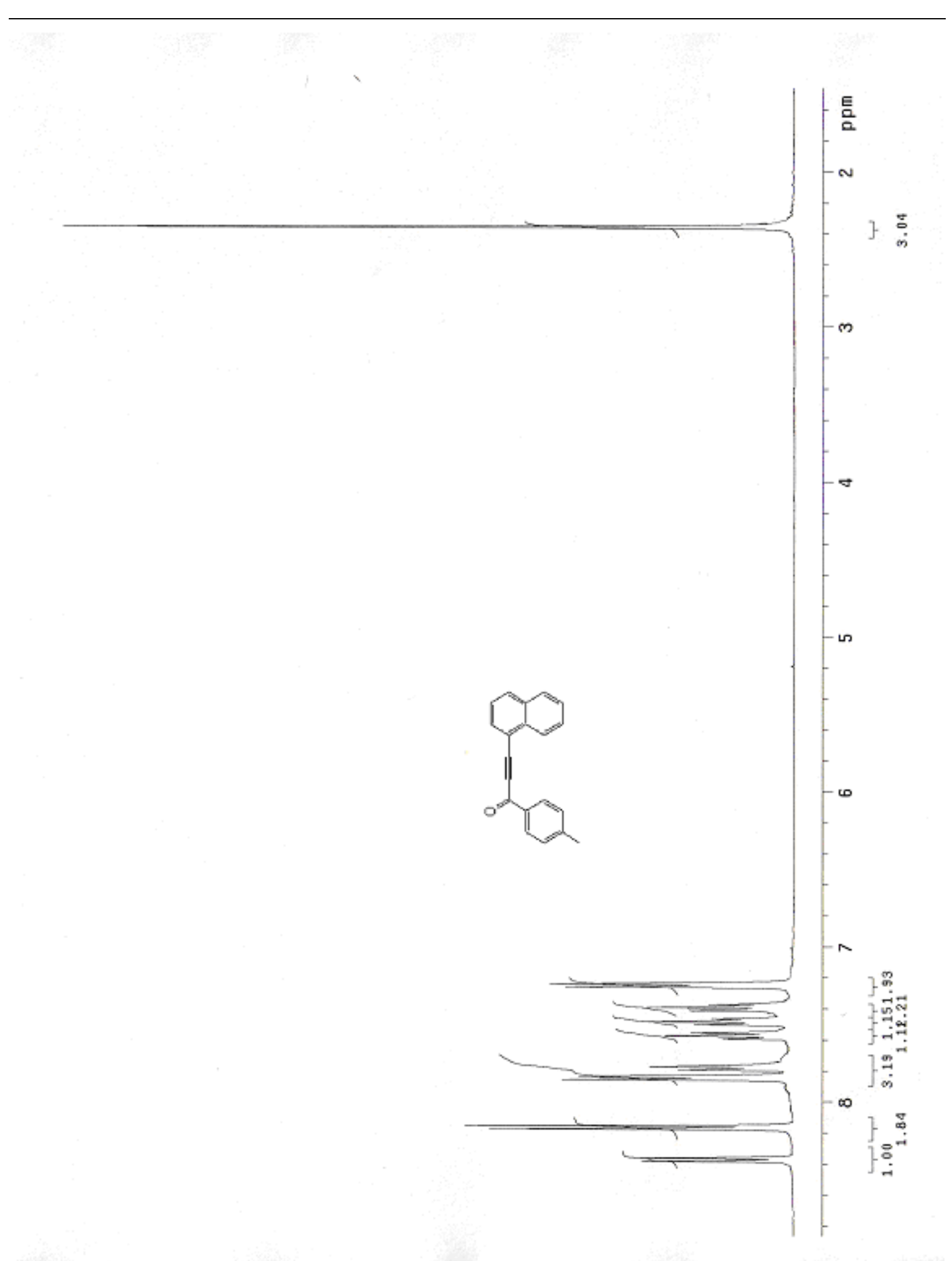

S-10 


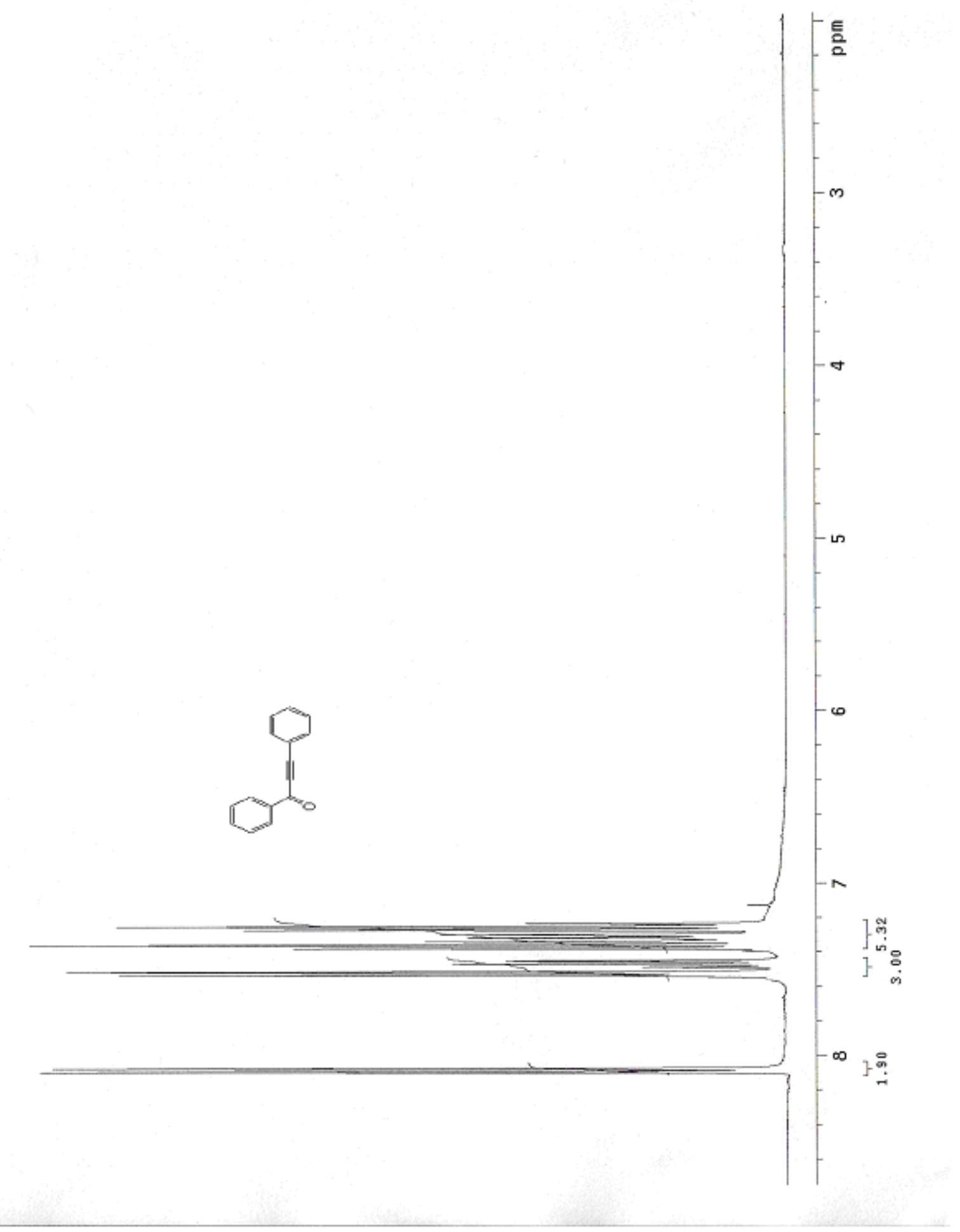

S-11 


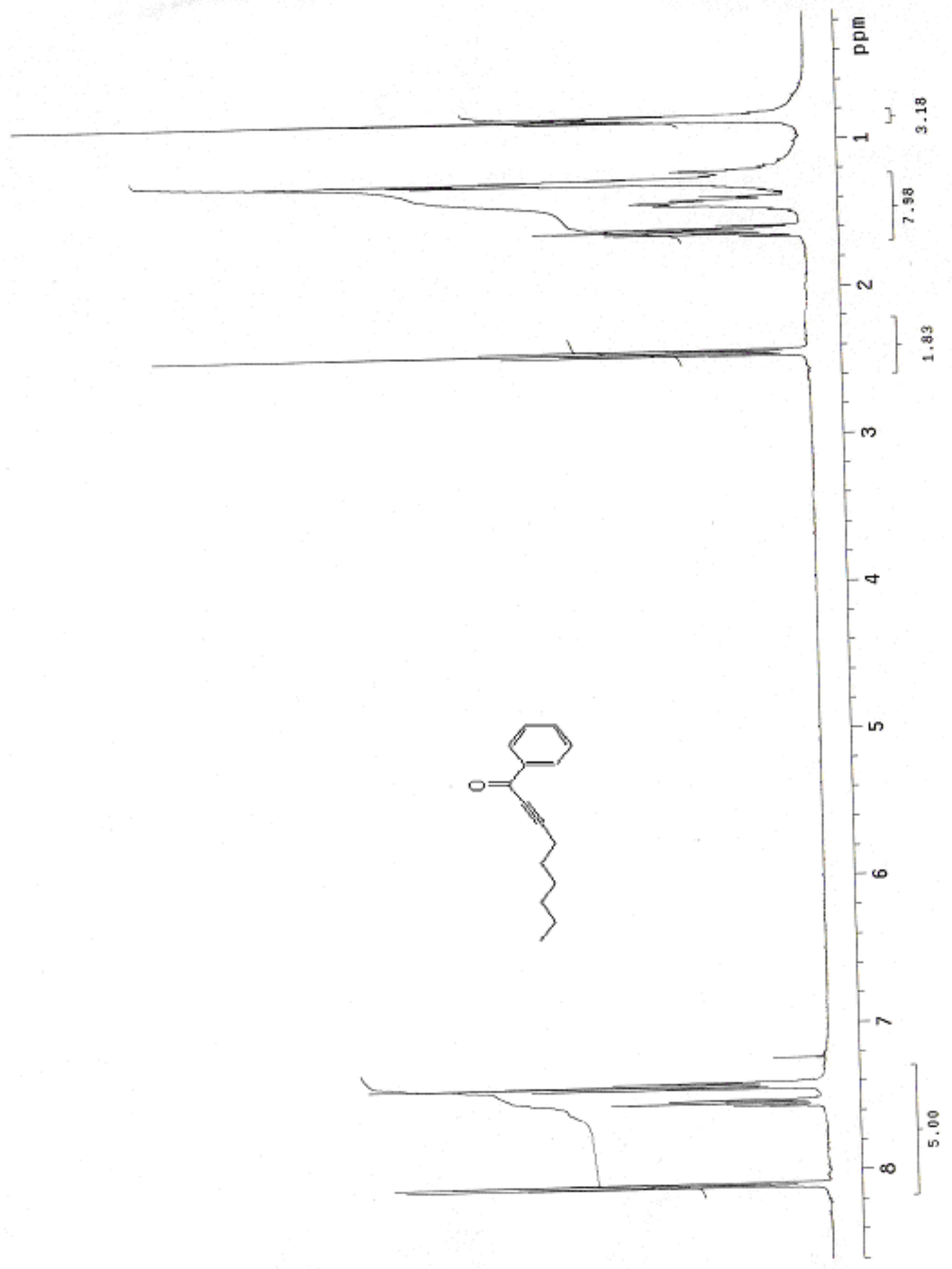

S-12 


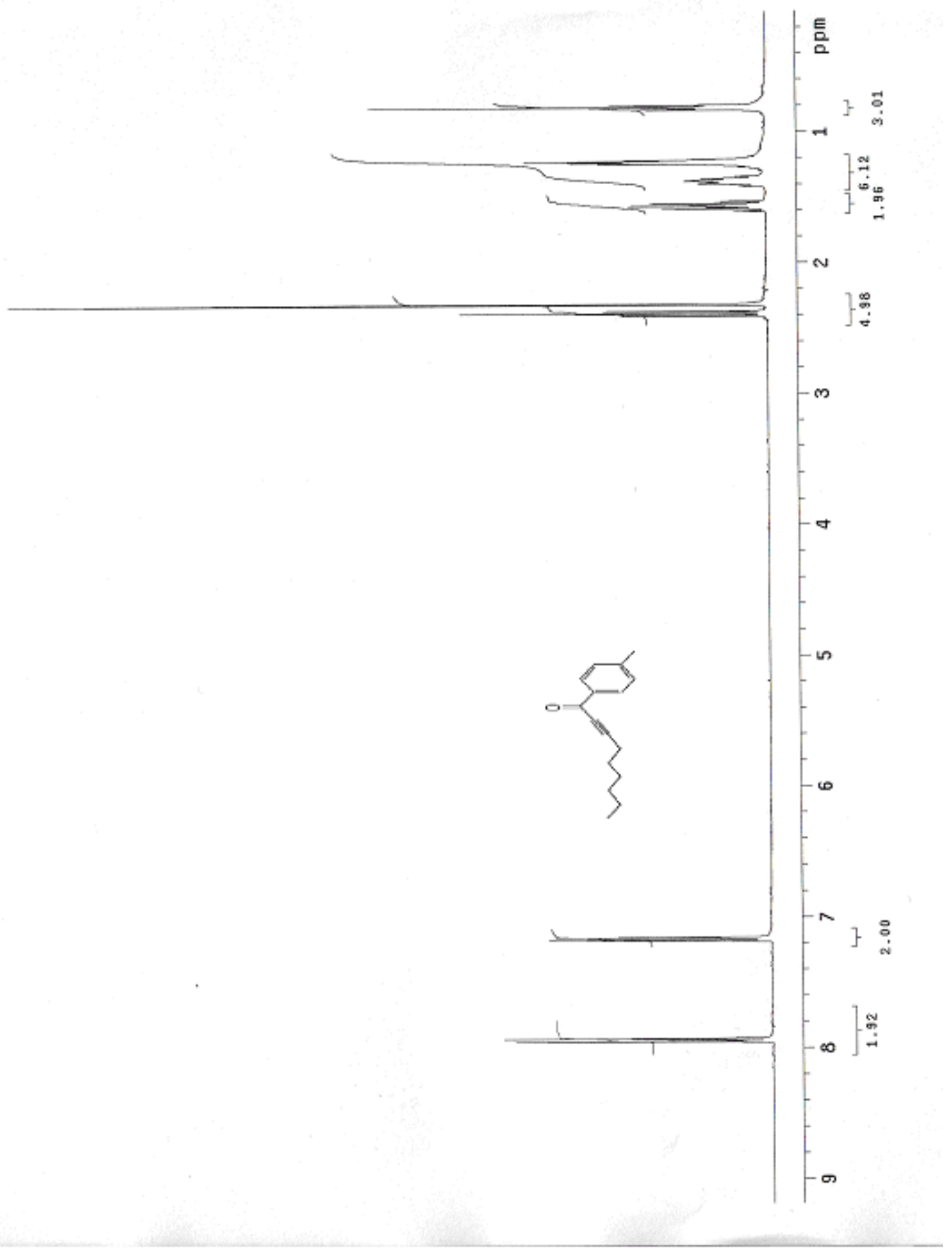

S-13 


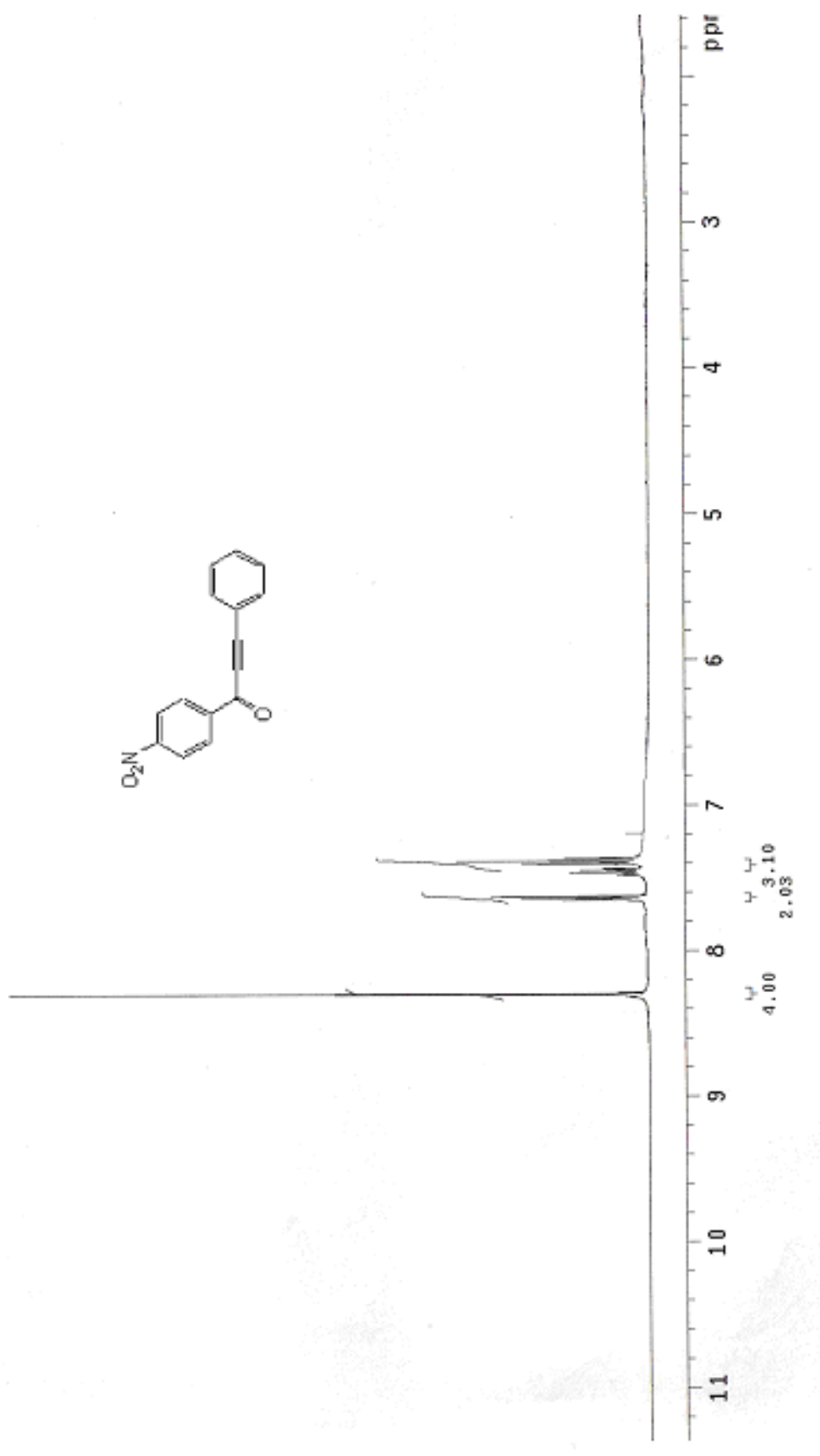

S-14 


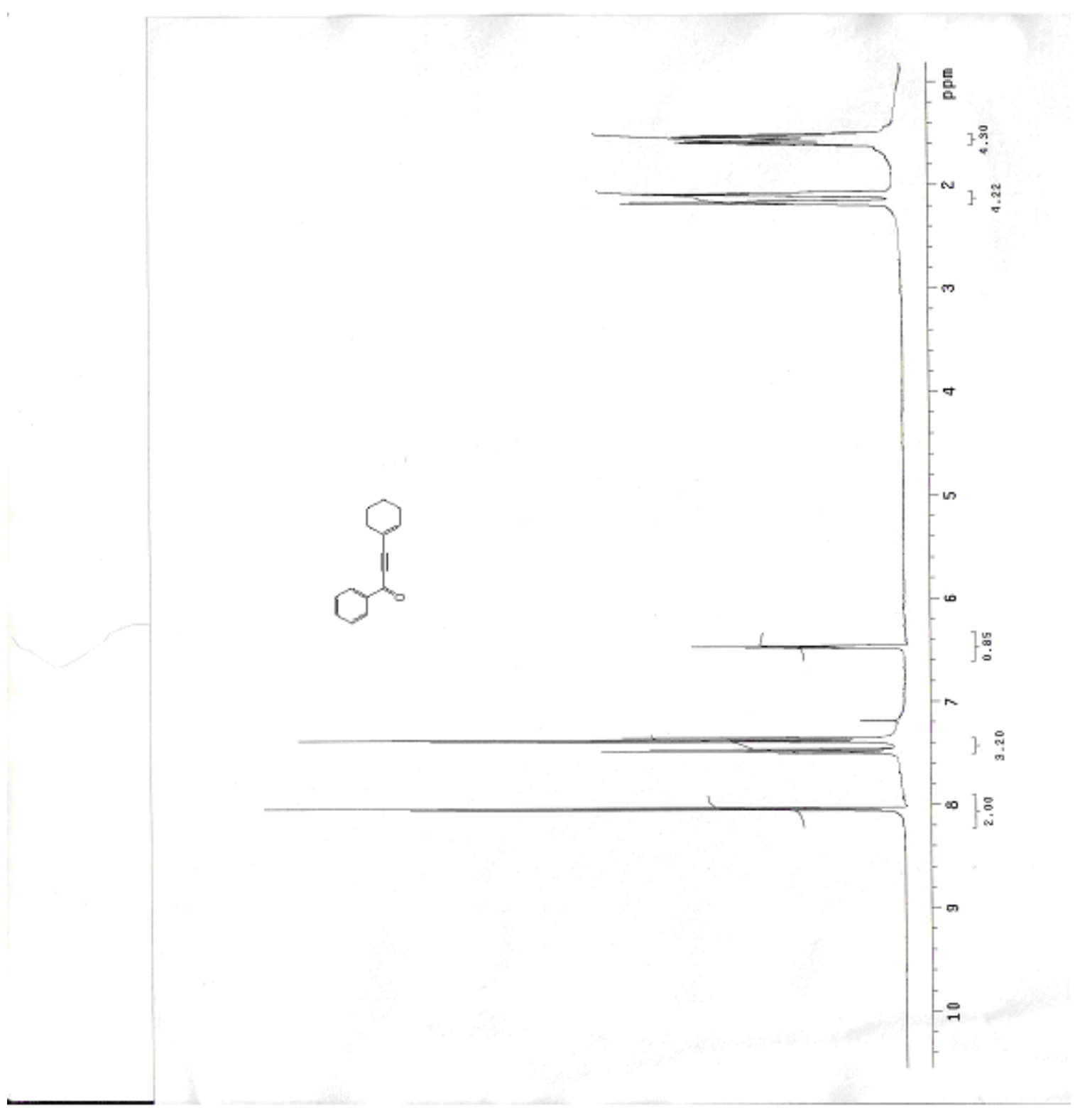

S-15 


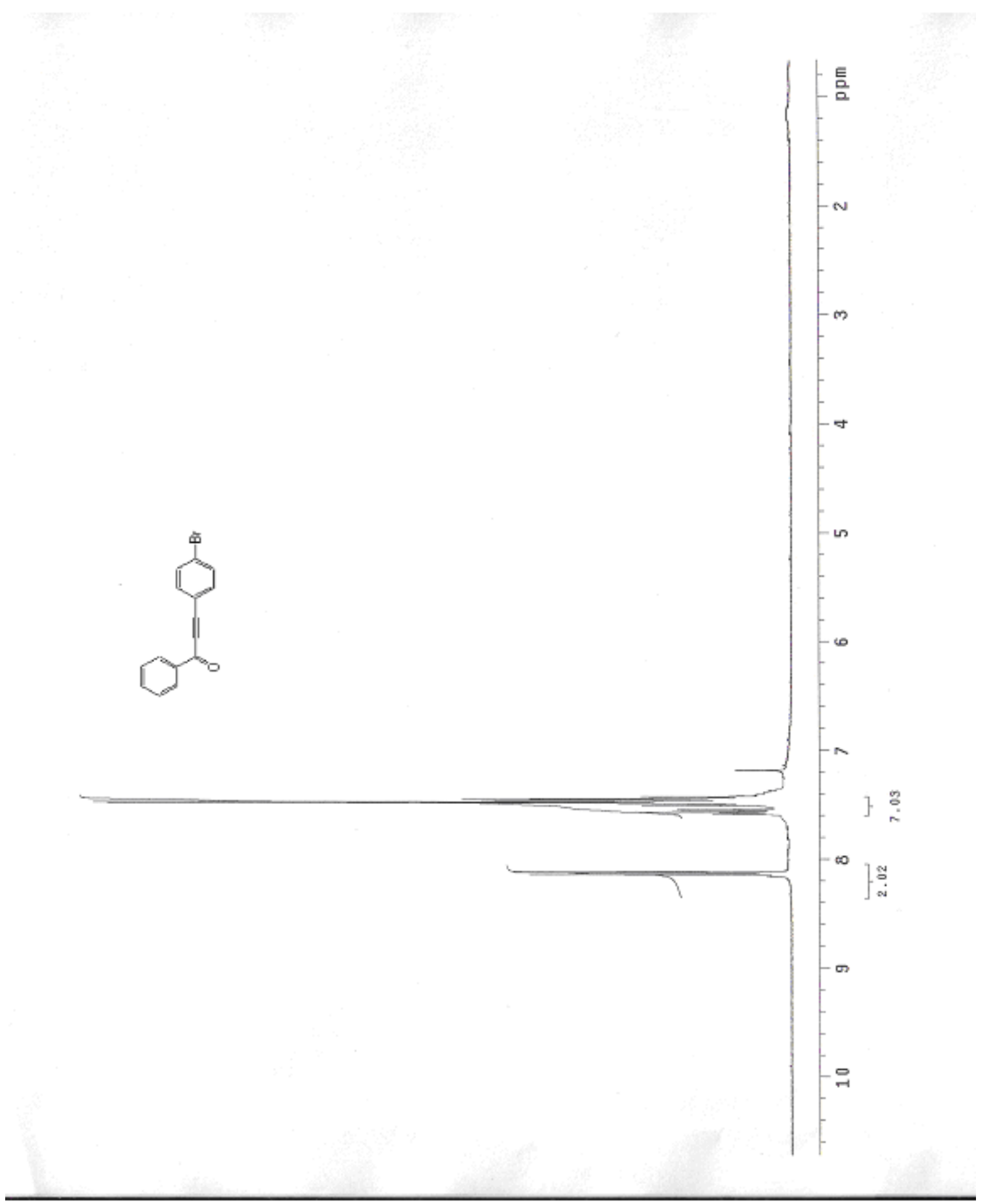

S-16 


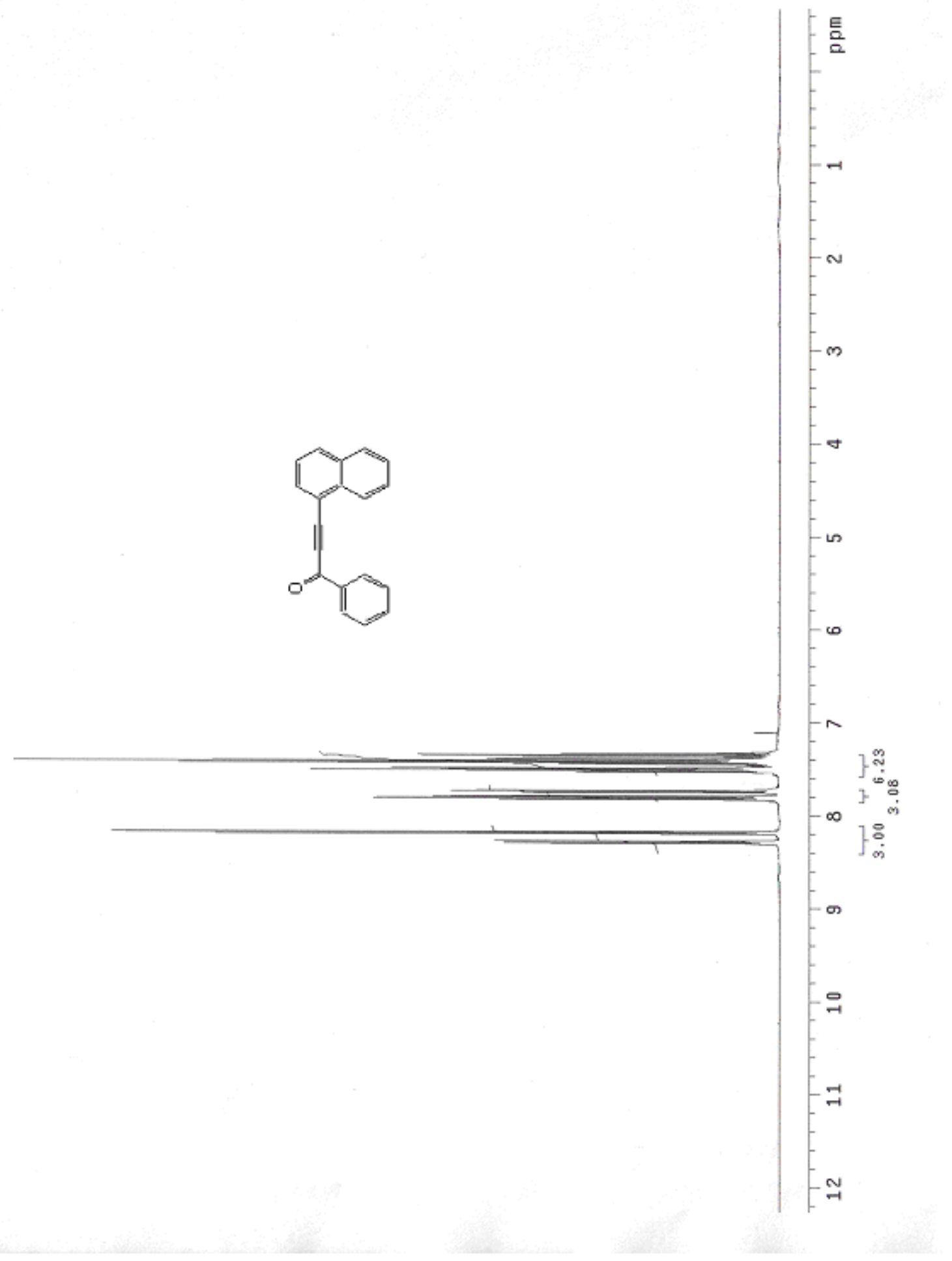

S-17 


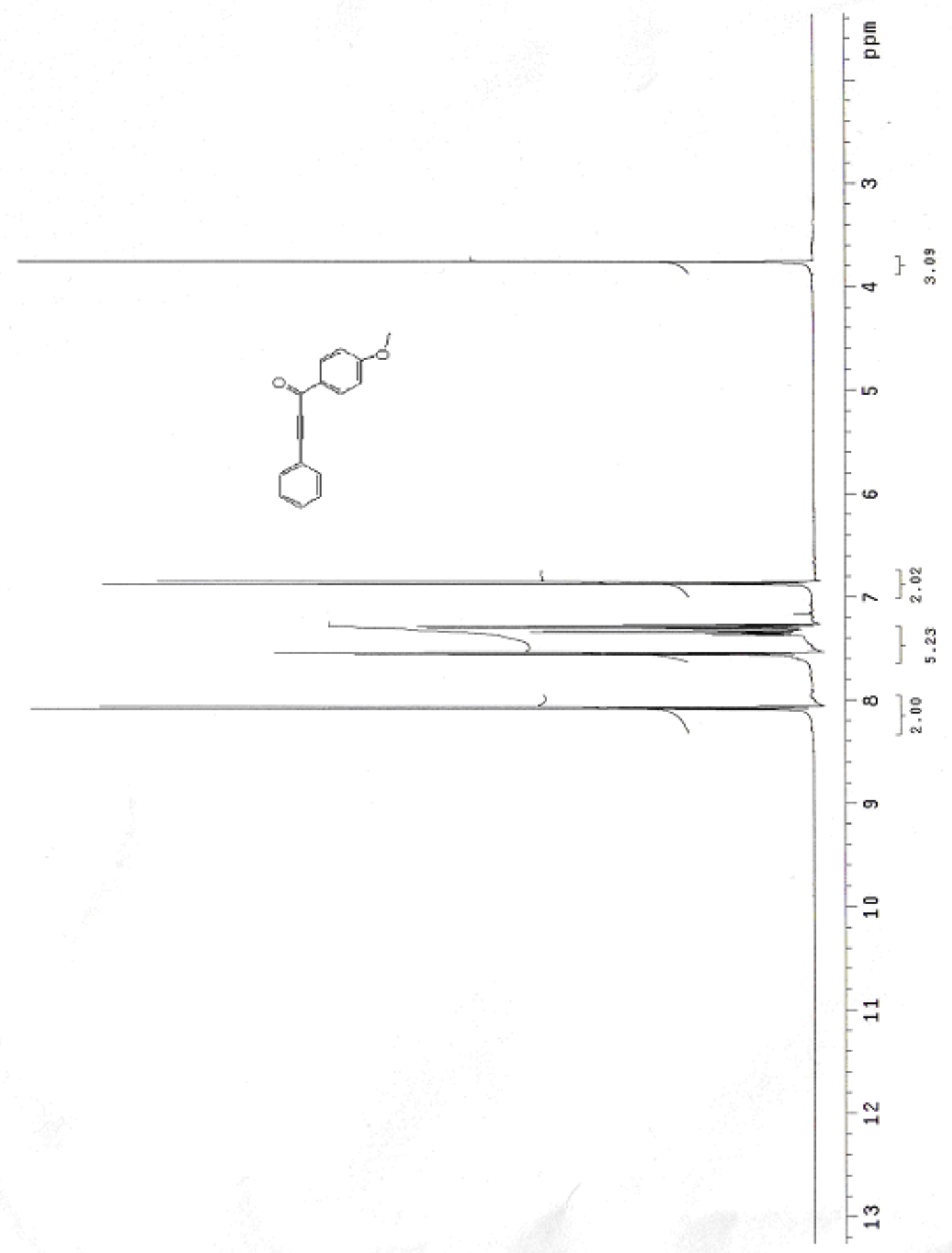

S-18 


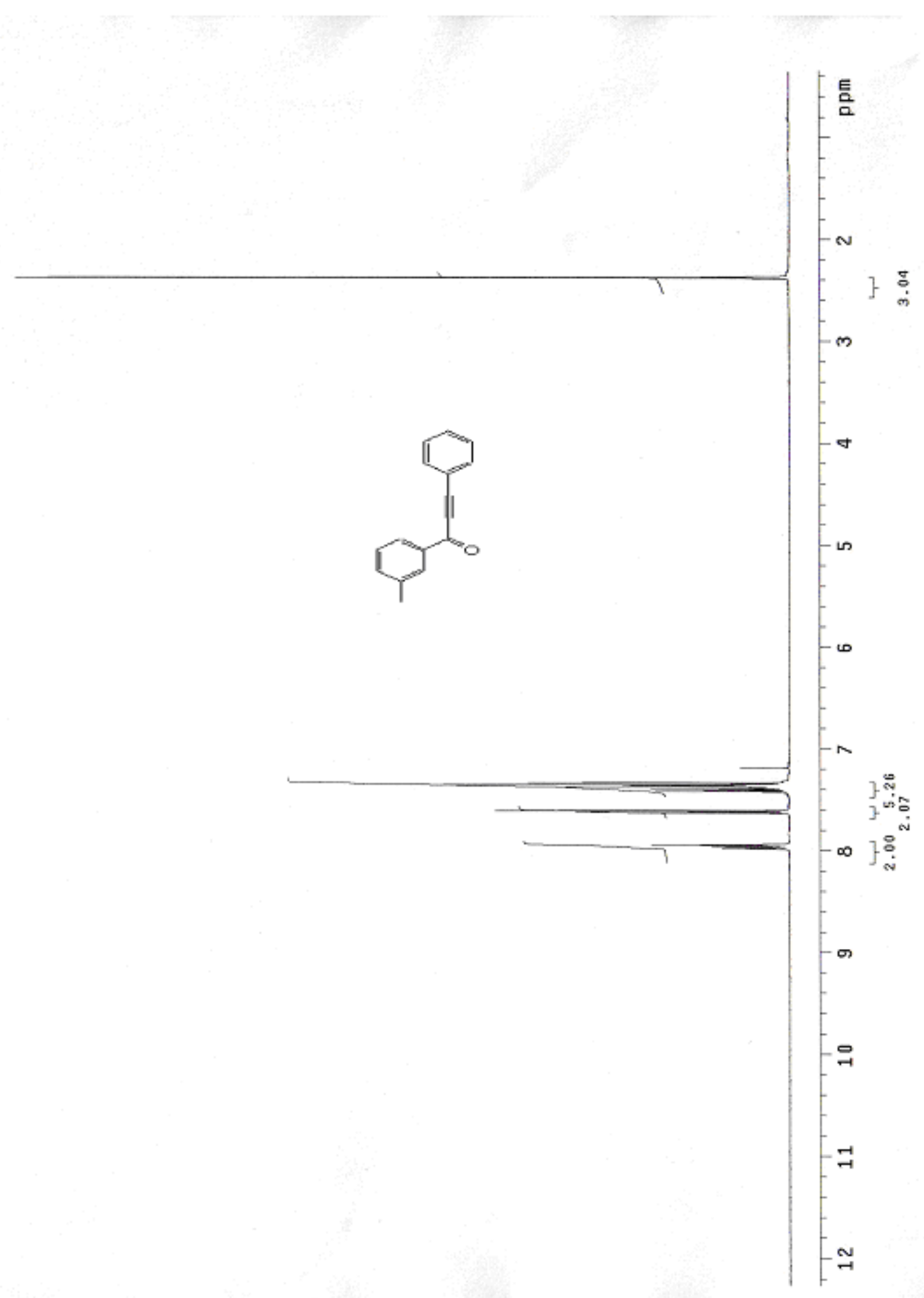

S-19 


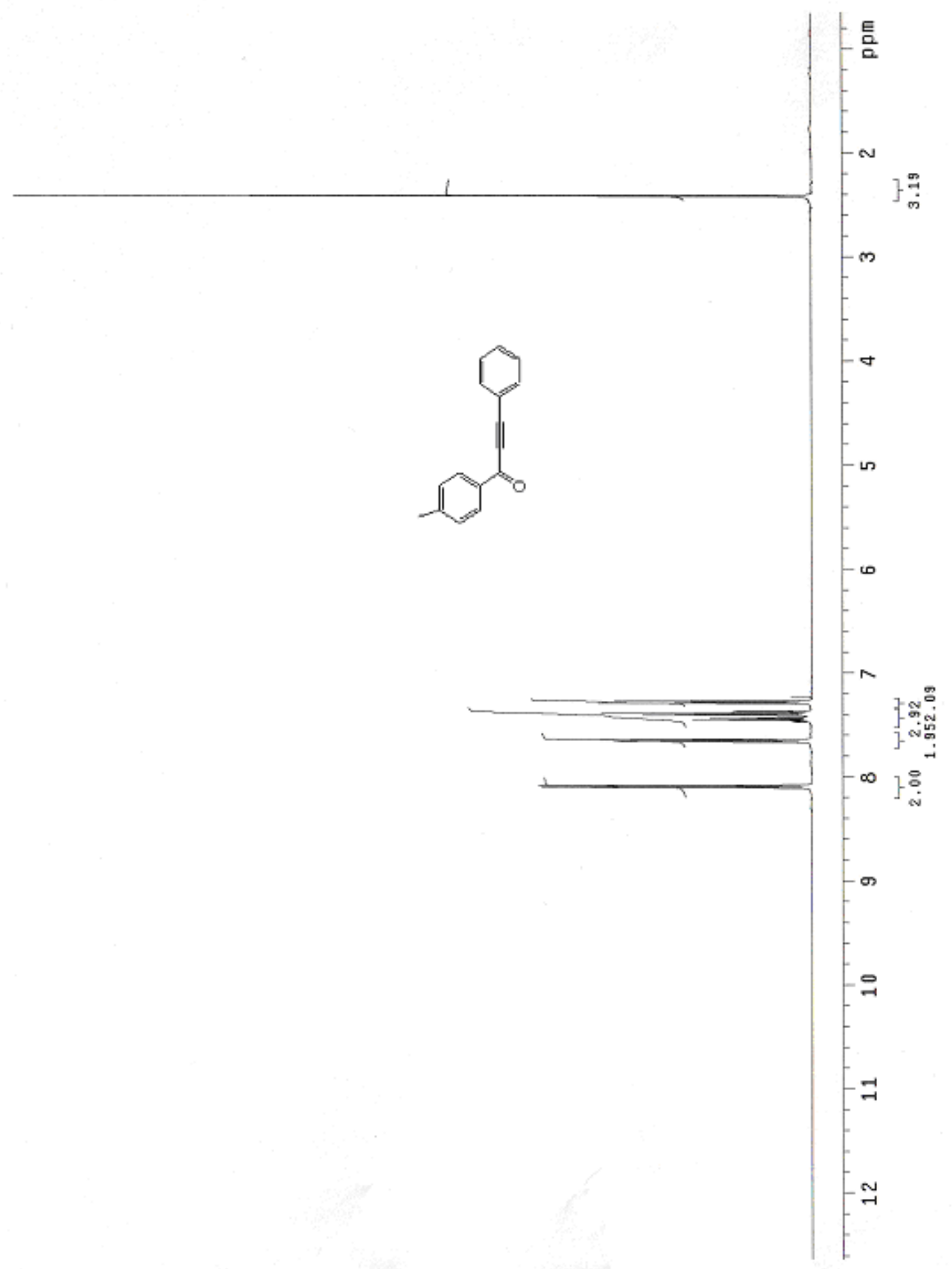

S-20 


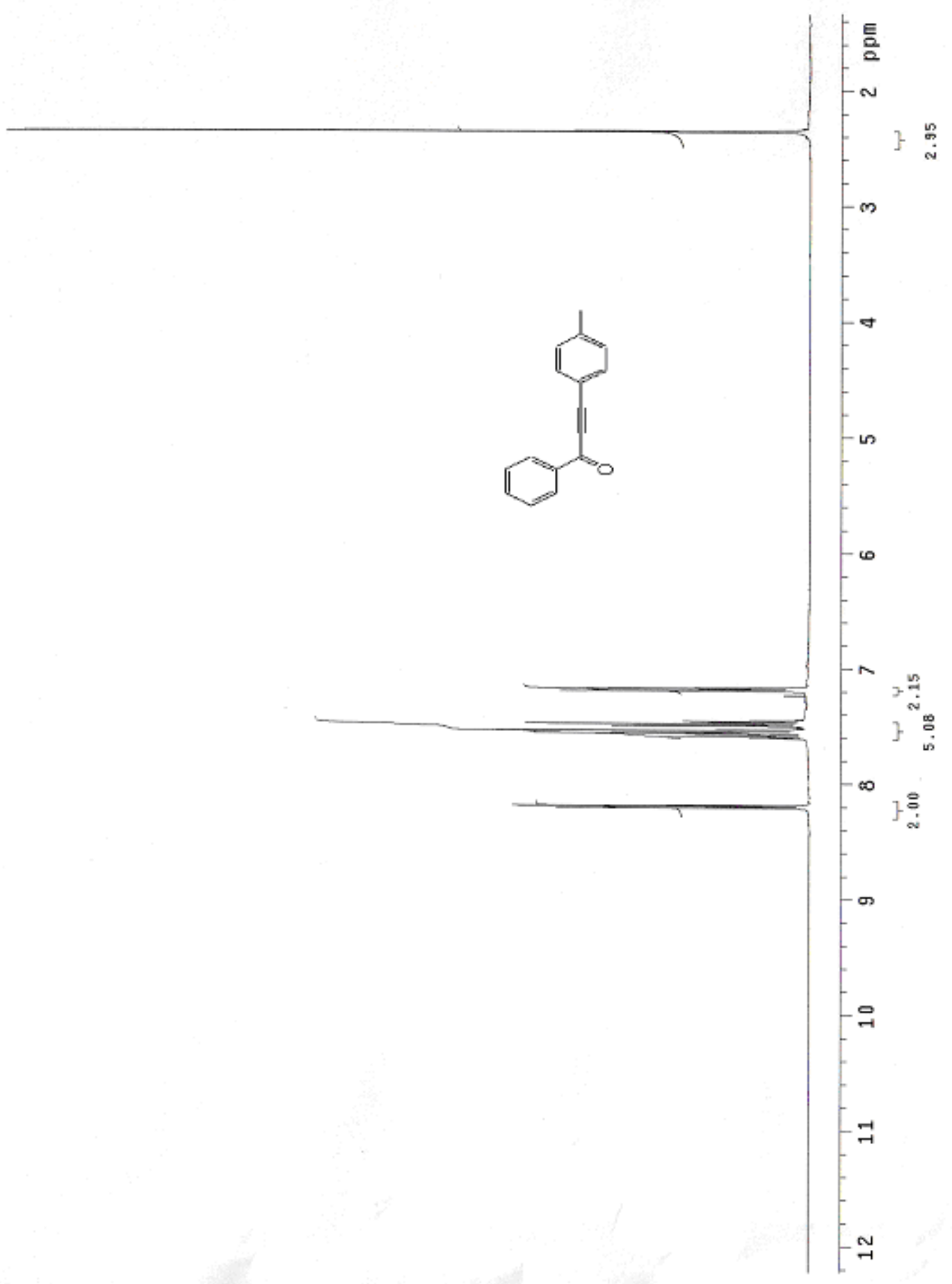

S-21 